\title{
Collision risk estimation on parallel airways
}

\author{
Qu Yuling ${ }^{1,2,}$, a , Chen Yanli $^{3}$, and Zhong Weiqing ${ }^{4}$ \\ ${ }^{1}$ College of Foundation Science, Harbin University of Commerce, Harbin Heilongjiang 150028, China \\ ${ }^{2}$ Business Administration Post Doctoral Mobile Station, Harbin University of Commerce, Harbin \\ Heilongjiang 150028, China \\ ${ }^{3}$ School of Management, Harbin University of Commerce, Harbin Heilongjiang 150028, China \\ ${ }^{4}$ School of Economics and Management, Jiangsu Maritime Vocational Institute, Nanjing Jiangsu 211100, \\ China \\ aquyuling2004@sina.com
}

Keywords: parallel airways, collision risk estimation, airspace planning, air traffic management

\begin{abstract}
In this paper, for purposes of airspace planning, by the theory of statistical-probabilistic and theory of traffic engineering a mathematical expression of the parallel airways based on the variable nominal distance and airspace planning parameters is given. By using the estimation formula proposed the average number of collisions between the parallel airways can be calculated and the impacts of airspace planning parameters on the collision risk can be shown. A method to characterize the collision risk when the aircraft are flying on parallel airways and the collision risk when the nominal distances on parallel airways are variable is given in the progress of airspace planning which may provide some theoretical basis for airspace planners to determine the lateral separation minimum, and may give some references basis for researchers of air traffic flow to study the capacity of airspace and air traffic controllers to keep airspace safe.
\end{abstract}

\section{Introduction}

In the field of air traffic management, the results of risk analysis will affect the actions performed of air traffic controllers because a main task of air traffic management is to contribute to risk reduction by keeping safe separation minima and administrations may use the methodology as a tool to estimate the collision risk in determining safe separation minima ${ }^{[1,2]}$. Every change of the operating procedure implementation in the progress of planning airspace and air routes is preceded by risk analysis in order to make the flight safe ${ }^{[3]}$. Airspace planners need to assess different scenarios for airspace development. Since aircraft safety level must be maintained when a new planning program is introduced in airspace, it is necessary to estimate the collision risk of this new planning program ${ }^{[2]}$, thus it is significant to integrate the collision risk estimation with airspace planning parameters in the revised airspace.

A collision risk model for long-range parallel air-routes over oceanic areas was given by Reich P. G. in 1966. Mr. Reich have studied the separation standards, collision risk and the quality of navigation which would be needed for safe reduction in standard ${ }^{[4]}$. The Reich model has a great influence on collision risk model. The problems of safe separations are resolved well by Reich and the model is applied extensively ${ }^{[5,6,7]}$. But the derivation of Reich model is a comparatively abstract one which does not make it easy to see what the key parameters are. Hsu researched the aircraft collision risk problem of intersecting air-routes for aircraft pairs flying on level air-routes at the same altitude with the invariable speed and both the Gaussian and the double double exponential model are used for the position error distributions to compute the aircraft overlap probability ${ }^{[8]}$. A Post-Reich' event model was developed by Brooker for lateral collision risk in air traffic track systems and applied to lateral collision in the North Atlantic Region track system, which resolves the problems of the Reich model ${ }^{[9]}$. It is a direct and concrete approach focusing on events, in contrast to Reich's 
synthetic methodology. Some researches about the collision risk based on event model are given ${ }^{[10,11]}$. In virtue of the model in reference[2], Han songchen and Qu yuling researched the collision risk model of terminal area airspace and around intersection of airways based on arrival time interval $^{[12,13]}$.

The researches above of the collision risk on parallel airways are mainly apt to invariable nominal distances. At the same time in considering the situation of stochastic aircraft flow, the existing collision models are apt to use the distribution function of initial nominal distance separation on parallel airways. Though in reference [2] the time variable $t$ is introduced in expressing the distance function, in the progress of derivation of collision probability the fixed time $t=T$ is used instead of the variable $t$. In contrast to the most exiting collision risk models which are mainly about the invariable nominal invariable, in this paper, the further research on collision risk based on the variable nominal invariable, time variable and initial time interval variable on parallel airways will be shown. Based on the references $[2,12,13]$, this paper is intended for use by airspace planners as a basis for changing separation minima and presents a mathematical expression for the analysis of collision risk applicable to airspace planning parameters and lateral separation of aircraft on parallel airways.

\section{Basic assumptions and definitions}

1) It is assumed that the aircraft are flying along a straight line flight path at the same nominal level on parallel airways.

2) Denote one airway by $K$ and another airway by $L$.

3) Denote the length of airway $K$ by $\operatorname{dis}_{K}$ and the length of airway $L$ by $\operatorname{dis}_{L}$.

4) Denote the starting point and stopping point of airway $K$ by $A$ and $B$ respectively. Denote the starting point and stopping point of airway $L$ by $C$ and $F$ respectively.

5) Aircraft 1is flying on the airway $K$ and the type is $i$, and aircraft 2 is flying on the airway $L$ and the type is $j . i=1,2, \cdots, k, \quad j=1,2, \cdots, l, k$ and $l$ is the number of types of aircraft on the airway $K$ and on the airway $L$ respectively.

6) It is assumed that the aircraft 1 arrives at the starting point $A$ earlier than the ircraft 2 arrives at the starting point $C$.

7) The time is $t=0$ when aircraft 2 arrives at point $C$.

8) Denote the time interval between aircraft 1 arrives at point $A$ and aircraft 2 arrives at point $C$ by $\Delta t$ and the time interval minimum by $\Delta t_{\min }$.

9) Denote nominal longitudinal direction by $x$, lateral direction by $y$, and vertical direction by $z$.

10) $\mathrm{D}$ enote the longitudinal distance between the starting point $A$ of airway $K$ and the starting point $C$ of airway $L$ by $d^{2} s_{x}$.

11) The units of this paper that haven’t been given are nautical for distance, hour for time and knot for speed.

\section{The collision risk estimation formula}

The number of aircraft involved in one collision is two. And the collision risk estimation on parallel airways will be shown. Lateral separation $S_{i j}^{y}(t, \Delta t)=S_{y}$ is a fixed value and vertical separation $S_{i j}^{z}(t, \Delta t)=0$ for a given planning program by supposing that a randomly chosen pair of aircraft is at the same level. Clearly $V_{i}(t+\Delta t)-V_{j} t \cos \theta-\eta \cdot d_{x}$ is longitudinal nominal distance which is the function of $t$ and varies with $t$, where, $\theta$ is $0^{\circ}$ when the aircraft on airway $K$ and the aircraft on airway $L$ fly in the same direction, and $\theta$ is $180^{\circ}$ when the aircraft on airway $K$ and the aircraft on airway $L$ fly in the opposite direction; $\eta$ is 1 when aircraft 1 is flying from $A$ to $B$ and $C$ is on the right side of $A$, and $\eta$ is -1 when aircraft 1 is flying from $A$ to $B$ and $C$ is on the left side of $A$; $V_{i}$ and $V_{j}$ is the nominal speed of aircraft 1 and aircraft 2, respectively.

Letting $\left|\chi_{i j}(t)\right|$ be the distance in the direction of $\chi$ at $t \in\left[0, t_{1}\right]$, then for any fixed 
$\Delta t \in\left[\Delta t_{\min }, d i s_{K} / V_{i}\right)$ and any fixed $t \in\left[0, t_{1}\right]$, where $\left.t_{1}=\min \left\{\operatorname{dis}_{K} / V_{i}-\Delta t, \operatorname{dis}_{L} / V_{j}\right\}\right)$, by the reference [13], we have

$$
\chi_{i j}(t)=\varepsilon^{\chi}+S_{i j}^{\chi}(t, \Delta t)
$$

Where, $\chi=x, y, z$, and $\varepsilon^{x}, \varepsilon^{y}, \varepsilon^{z}$ is the longitudinal, lateral and vertical error variable respectively. In this paper, as in Doc9689-An/953 ${ }^{[2]}$, an equivalent geometry that is a cylinder of $\lambda_{x y}$ radius and height $2 \lambda_{z}$ is used to characterize one aircraft.

Let $S_{i j}^{x}(t, \Delta t)=V_{i}(t+\Delta t)-\cos \theta V_{j} t-\eta \cdot d_{x}, S_{i j}^{y}(t, \Delta t)=S_{y}, S_{i j}^{z}(t, \Delta t)=0$. By the reference [2], for any fixed $\Delta t \in\left[\Delta t_{\min }, d i s_{K} / V_{i}\right)$ and any fixed $t \in\left[0, t_{1}\right]$ we have the collision probability of two aircraft as follow

$$
\begin{aligned}
P_{i j}\left(t, \Delta t ; S_{y}\right)= & P_{i j}^{x}\left(t, \Delta t ; S_{i j}^{x}(t, \Delta t)\right) \times P_{i j}^{y}\left(t, \Delta t ; S_{y}\right) \\
& \times P_{i j}^{z}(t, \Delta t ; 0) \times\left(1+\pi \lambda_{x} \bar{z} \mid /\left(4 \lambda_{z} V_{r e l}\right)\right) .
\end{aligned}
$$

where, $P_{i j}^{\chi}\left(t, \Delta t ; S_{i j}^{\chi}(t, \Delta t)\right)=P\left(\left|\chi_{i j}(t)\right| \leq \lambda_{\chi}\right)$ and $\lambda_{x}, \lambda_{y}, \lambda_{z}$ is average aircraft height, average aircraft wingspan and average aircraft height respectively; $V_{\text {rel }}$ is the relative speed of the two aircraft conditional on a horizontal overlap taking place; $|\bar{z}|$ is the relative speed of the two aircraft conditional on vertical direction.

Furthermore, we can have that the average number of aircraft pairs formed between one aircraft typed $i$ on the airway $K$ and the aircraft on the airway $L$ in the time length $t_{1}$ is $N P_{i}$. Thus, by the reference [13], when aircraft is flying on the airway $K$ for an hour, the average number of collisions coming from the aircraft on the airway $L$ can be expressed as

$$
\begin{aligned}
C R=2 \sum_{i=1}^{k} \sum_{j=1}^{l} & \left(p_{K i} p_{L j} N P_{i} \frac{V_{i}}{d i S_{K}} \int_{\Delta t_{\min }}^{d i s_{K} / V_{i}} g(\Delta t)\right. \\
& \left.\times \frac{1}{t_{1}} \times \int_{0}^{t_{1}} P_{i j}\left(t, \Delta t ; S_{y}\right) d t d \Delta t\right) .
\end{aligned}
$$

Where, $g(\Delta t)$ is the density function of $\Delta t ; p_{K i}$ is the proportion of the aircraft type $i$ in the total aircraft on the airway $K$ and $p_{L j}$ is the proportion of the aircraft type $j$ in the total aircraft on the airway $L$.

\section{The calculation and analysis of collision risk}

\subsection{Definitions of parameter values}

In the following sections, navigation error and speed error are used to calculate collision risk. Generally speaking, the core distribution is Gaussian for some types of errors, some researchers assume that all navigation and tracking errors are Gausian ${ }^{[14,15,16]}$. Because the convolution of two Gaussian densities is another Gaussian density, which avoids the need for numerical convolution calculations, for the consideration of mathematical simplicity, in this paper Gaussian distribution model is selected for describing position errors. Then we have

$$
\begin{aligned}
& \varepsilon^{x}=\varepsilon_{1 n}^{x}+\varepsilon_{1 v}^{x}(t+\Delta t)+\left(-\varepsilon_{2 n}^{x}-\varepsilon_{2 v}^{x} t\right) \cos \theta \\
& \varepsilon^{y}=\varepsilon_{1 n}^{y}+\varepsilon_{1 v}^{y}(t+\Delta t)-\left(\varepsilon_{2 n}^{y}+\varepsilon_{2 v}^{y} t\right) \cos \theta \\
& \varepsilon^{z}=\varepsilon_{1}^{z}-\varepsilon_{2}^{z}
\end{aligned}
$$

Where, $\varepsilon_{m n}^{x}$ and $\varepsilon_{m n}^{y}(m=1,2)$ is the longitudinal and lateral navigational error variable of aircraft $m$ respectively; $\varepsilon_{m v}^{x}$ and $\varepsilon_{m v}^{y}$ is the longitudinal and lateral speed error variable of aircraft $m$ respectively; $\varepsilon_{m}^{z}$ is the vertical error variable of aircraft $m$.

So $\varepsilon_{m \gamma}^{\tau} \sim N\left(0, \sigma_{m \gamma}^{\tau}\right) \quad(\tau=x, y, \gamma=n, v), \varepsilon_{m}^{z} \sim N\left(0, \sigma_{m}^{z}\right), \quad m=1,2$. . In practical applications, the actual 
distribution of deviations is likely to be acquired by the more information and data amassed in the concrete airspace. The situations of other distribution functions of deviations can be discussed by using the same method. It is assumed that errors are independent.

Computations are carried out for RNP (required navigation performance) value-4 in the following segments. The density function of navigation error distribution in the longitudinal direction and lateral direction can be specified on the basis of the RNP type of the airspace. So the standard deviation of navigation error may be determined by the RNP-n, i.e. the standard deviation of navigation error can be given by the value 4 of RNP multiplied by the factor $1 / 1.96$. The standard deviation of speed error is 5.82 nautical mile per hour and $\sigma_{m}^{2}=35 / 1852(m=1,2)$.

By assuming that the flow of along airways is Poisson distributed and the flow rate of airway $L$ is $\lambda_{L}$, we can have the average number of aircraft on airway $L$ in the time length $t_{1}$ is $\sum_{k=1}^{\lambda_{L}}\left(\left(\lambda_{L} t_{1}\right)^{k} e^{-\lambda_{L} t_{1}} /(k-1) !\right)$.

Furthermore $\Delta t$ is assumed to be uniform on $\Delta t \in\left[\Delta t_{\min }, d i s_{K} / V_{i}\right)$. The density function of $\Delta t$ can be given by $g(\Delta t)=1 /\left(\operatorname{dis}_{K} / V_{i}-\Delta t_{\min }\right), \quad \Delta t \in\left[\Delta t_{\min }, \operatorname{dis}_{K} / V_{i}\right)$.

The collision risk estimation requires values of some basic parameters used in the calculation are presented in Table 1.

Table 1. Basic collision risk estimation parameter values

\begin{tabular}{ccccccc}
\hline Parameter & $\lambda_{x} / \mathrm{n}$ mile & $\lambda_{y} / \mathrm{n}$ mile & $\lambda_{z} / \mathrm{n}$ mile & $V_{\text {rel }} / \mathrm{kn}$ & $\overline{|\bar{z}|} / \mathrm{kn}$ & $\Delta t_{\min } / \mathrm{h}$ \\
\hline Value & 0.0417 & 0.0417 & 0.0114 & 35 & 1.5 & 0 \\
\hline
\end{tabular}

\subsection{The collision risk calculation of parallel airways}

(1) For $\eta=-1, \lambda_{c}=6, S_{y}=12, d_{x}=3, \theta=0^{\circ}$, by Eq.3, we can have that the average number of collisions for different $d i s_{K}$ and $d i s_{L}$, see Table 2.

Table 2. Results for different $d i s_{K}$ and $d i s_{L}$

\begin{tabular}{ccccc}
\hline $\mathrm{dis}_{K} / \mathrm{n}$ mile & dis $_{L} / \mathrm{n}$ mile & $V_{i} / \mathrm{kn}$ & $V_{j} / \mathrm{kn}$ & $C R$ \\
\hline 300 & 280 & 480 & 480 & $9.5924 \times 10^{-9}$ \\
200 & 150 & 480 & 480 & $3.6319 \times 10^{-9}$ \\
100 & 150 & 480 & 480 & $1.8330 \times 10^{-9}$ \\
100 & 100 & 480 & 480 & $1.8330 \times 10^{-9}$ \\
300 & 280 & 600 & 600 & $4.5743 \times 10^{-9}$ \\
200 & 150 & 600 & 600 & $1.9959 \times 10^{-9}$ \\
100 & 150 & 600 & 600 & $1.6469 \times 10^{-9}$ \\
100 & 100 & 600 & 600 & $1.6469 \times 10^{-9}$ \\
300 & 298 & 480 & 480 & $9.5924 \times 10^{-9}$ \\
200 & 202 & 480 & 480 & $3.6319 \times 10^{-9}$ \\
100 & 102 & 480 & 480 & $1.8330 \times 10^{-9}$ \\
\hline
\end{tabular}

The results given in the Table 2 show the influences of the length of airway and speeds of aircraft on the collision risk $C R$. By the results of calculation we found that the longer $\operatorname{dis}_{K}$, the greater collision risk conditional on other parameter values fixed and the smaller speed the greater collision risk when other parameter values are fixed. By the model proposed in this paper we can analyze the main reasons as follows: The first main reason is that when other parameter values are fixed the longer $\mathrm{dis}_{K}$, the longer flight time on airway $K$ which causes the greater extrapolation errors; The second main reason is that the smaller average speed, the longer flight time conditional on other parameter values fixed. By the analysis we can have the conclusion that the collision risk is also increasing with the increased the proportion of aircraft type which is of the smaller speed when other parameter values are fixed. 
(2) The collision risk which there are two types of aircraft on parallel airways is considered. Consider the collision risk where we have three cases:1) Let the speed of aircraft be 480and480 , respectively; (2) Let the speed of aircraft be 600and600, respectively; (3) Let the speed of aircraft be 480and600 , respectively, and let the proportion be $95 \%$ and $5 \%$, respectively. Then for $\eta=1, \lambda_{c}=6, d_{x}=3, \operatorname{dis}_{K}=300, \operatorname{dis}_{L}=280, \theta=0^{\circ}$, by Eq.3, we can have that the average number of collisions for different $S_{y}$, see Fig. 1 .

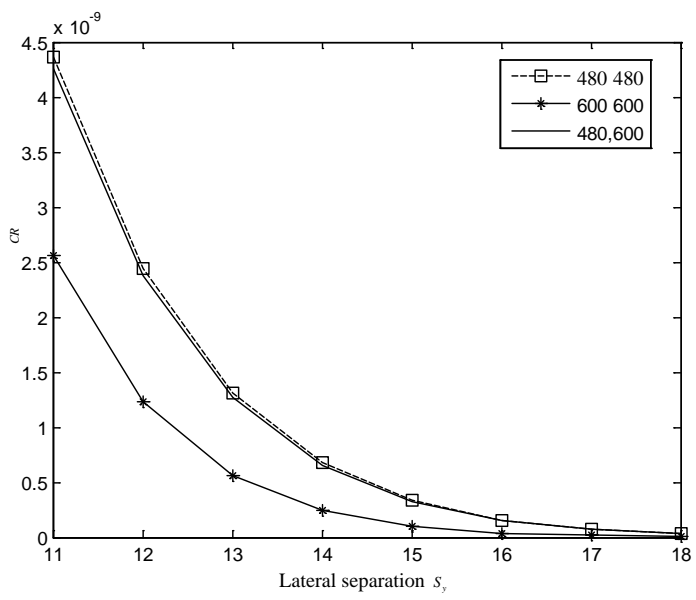

Fig.1. The relation between the number of collisions and lateral separation $S_{y}$

The results of Fig. 1 show that collision risk $C R$ varies with the lateral separation $S_{y}$. The collision risk will decrease with the increased lateral separation $S_{y}$. It is a very obvious result the wider separation, the less collision risk.

(3) Let the speed of aircraft be 480 and 480, respectively. For $d i s_{K}=180$, dis $s_{L}=180, S_{y}=12$, by Eq.3, we can have that the average number of collisions $C R$ when $\eta, d x, \lambda_{c}$ and $\theta$ take different values, see Table 3.

Table 3. $C R$ for different $\eta, d x, \lambda_{c}$ and $\theta$

\begin{tabular}{ccccc}
\hline$\eta /$ n mile & $d x /$ n mile & $\lambda_{c} /$ number of flight $/ \mathrm{h}$ & $\theta /\left({ }^{\circ}\right)$ & $C R$ \\
\hline-1 & 3 & 6 & 180 & $1.0693 \times 10^{-9}$ \\
1 & 20 & 6 & 180 & $1.8084 \times 10^{-9}$ \\
-1 & 10 & 10 & 0 & $4.8963 \times 10^{-9}$ \\
\hline
\end{tabular}

Table 3 shows the results of the estimation associated with variations of $\eta, d x, \lambda_{c}$ and $\theta$. The impact of parameter values on the collision risk can be seen in Table 2 and Table3. From results given in Table2 and Table 3, it is possible to calculate, for some given parameter values in the progress of airspace planning, the average number of collisions on parallel airways.

\section{Conclusions}

1) The collision risk when the nominal distances between aircraft are variable on parallel airways can be characterized by the method proposed in this paper. This paper describes the methodology to determine lateral separation minima for use on parallel airways. These theories could provide some references for planners to set the lateral separation and the reporting interval.

2) A method to research the collision risk based on some airspace planning parameters on parallel airways is given. Airspace planners need to estimate the collision risk when some related parameter values change in different scenarios in the progress of the airspace planning. The methodology presented in this paper provides a framework by which airspace characteristics, the number of flight per hour, speeds of aircraft, the proportion of the aircraft type and RNP which will give some 
theoretical basis for airspace planners to determine related parameter values and then determine lateral safe separation minima for parallel airways operations.

\section{Acknowledgements}

This paper is supported by HeiLong Jiang Province Education Foundation (12541190).

\section{References}

[1] International Civil Aviation Organization: Safety Management Mannual, Doc9859-An/460, third ed (International Civil Aviation Organization, Montreal, 2012).

[2] International Civil Aviation Organization: Mannual on airspace planning methodology for the determination of separation minima, Doc9689-An/953 (International Civil Aviation Organization, Montreal, 1998).

[3] J. Skorupski: submitted to Safety science (2016).

[4] P. G. Reich: submitted to Journal of Navigation (1966).

[5] Han Songchen, Pei Chenggong and Sui Dong: submitted to Acta Aeronautica ET Astronautica Sinica (2006).

[6] Zhang Zhaoning, Zhang Xiaoyan and Li Dongbin: submitted toJournal of Traffic and Transportation Engineering (2007).

[7] Zhu Daiwu, Li Chenlu and Chen Jiuhao: submitted to Aeronautical Computing Technique (2017).

[8] [9] D. A. Hsu: submitted to Journal of Navigation (1981).

[9] P. Brooker: submitted to The Journal of navigation (2003).

[10] Zhang Zhaoning and SHI Ruijun: submitted to China Safety Science Journal (2015).

[11] Zhao Zhenwu, Zhang Wei and Tang Yuli: Submitted to China Safety Science Journal (2016).

[12] Han Songchen, Qu Yuling, Sun Fanrong and Zhu Xinping: submitted to Journal of southwest jiaotong university (2013).

[13] Qu Yuling, Huo Zhiqin, Li chong, et al: submitted to China Safety Science Journal (2017).

[14] H. J.Rome, Proceeding of the institute of navigation technical meetion, Santa Barbara Conference 213-222 (1988).

[15] L. M. B. C.Campos: submitted to Journal of Aircraft (2001).

[16] L. M. B. C. Campos and J. M. G. Marques: submitted to Journal of Aircraft (2007). 